\title{
Triphasic CT Liver Characterization and Color Data Fusion
}

\author{
Silvana G. Dellepiane ${ }^{1}$, Mahdieh Khalilinezhad ${ }^{2}$, Roberta Ferretti ${ }^{3}$ \\ DITEN, Dipartimento di Ingegneria Navale, Elettrica, Elettronica e delle Telecomunicazioni, \\ Universita’ Degli Studi Di Genova, Italy ${ }^{1,2,3}$
}

\begin{abstract}
The aim of the present work is the analysis and mining of the informative content related to pathological liver tissues, when acquired by triphasic CT, with the proposal of a data-fusion approach, which is able to visualize and show up such a content in the best way as a support to the medical diagnosis. Since the huge amount of CT volumes to be analyzed in a limited time is the major cause of sensitivity loss during the diagnosis process, a better chance of detection and localization of the pathology can be derived from the method here proposed. This method can be a valid support to the current medical practice, even in the cases where pathology is at the very early stage and has a large probability to be missed by a visual inspection. As expected when analyzing the three phase volumes, one can note that the injection of a contrast agent causes significant changes in the radiological finding for both pathological and healthy parts of the liver. Thanks to a specific statistical analysis performed on a training dataset of real cases, the described study was focused on the characterization of hepatocellular carcinoma (HCC) tumor tissues and liver tissues.

In order to detect and discriminate tumor from liver parenchyma, we here propose using both steady-state and dynamic features. Some common patterns have been observed suggesting rules, which have been confirmed by radiology specialists. Based on the rules and the best discriminant power of some of the characterizing features, a new color data fusion approach is then proposed and discussed which improves the mass visibility while increasing contrast with respect to surrounding parenchyma.
\end{abstract}

Keywords: Triphasic CT, tissue characterization, feature analysis, data fusion, color distance.

\section{INTRODUCTION}

In general, lesion vascularity is fundamental when dealing With reference to the triphasic CT modality, this work has with liver pathologies and dynamic triphasic CT imaging two main objectives:

has recently acquired a strong relevance, since it allows - To explore a given dataset of real HCC cases in order to

the observation of arterial, portal-venous, and equilibrium discover the most substantial information content;

phases.

Hypervascular masses (which cover more than $75 \%$ of liver tumors [11]) have increased hepatic artery coefficients compared to the liver [1] and show hyper enhancement in the arterial phase. In the successive phases, when the enhancement of the surrounding liver overtakes that of the hypervascular mass, this finding is referred to as "negative enhancement," or washout [2]. Its presence favors a malignant diagnosis; its absence suggests benignancy [2].

As a consequence, the differential diagnosis is based on the observed arterial phase enhancement and the presence or absence of washout.

According to the new recommendations of the American Association for the Study of the Liver Diseases (AASLD), for lesions $\geq 1 \mathrm{~cm}$, characteristic findings with arterial hypervascularity and venous washout, in a dynamic 4 phases (unenhanced, arterial, venous and delayed) multidetector CT or MRI, is diagnostic for Hepatocellular carcinoma (HCC), without biopsy, in cirrhotic liver and in patients with chronic hepatitis B $[3,4]$.

In the current medical practice, the huge amount of dynamic tomographic volumes to be analysed in a limited time is one of the major reasons for sensitivity loss during the diagnosis process.

- To propose a new integrated visualization which increases the possibility of detecting the possible lesion in the earliest phase, before it reaches a size where the chance of cure is too weak.

To this aim, a quantitative statistical analysis has been performed dealing both with steady-state and temporal features. Some relevant rules that allow discriminating between healthy and pathological tissues have been discovered and drive the criteria for the subsequent data fusion step.

Based on such rules, a new color data-fusion approach is proposed and applied, able to visualize and show up the informative content in the best possible way, thus supporting medical screening and diagnosis.

After the description of the training set, Section II explains the performed statistical analysis starting from steady-state features. A few meaningful rules are proposed which represent the basis for the subsequent Color Data-Fusion Section (Section III). The experimental work is reported in Section IV, followed by the Conclusion Section.

All the analysis and processing steps are described in the next sections with the help of a specific case study that is particularly monitored and deeply examined. Surprisingly, the choice of the criteria that generate the best color data fusion result, based on the statistical analysis of the most 
International Journal of Advanced Research in Computer and Communication Engineering

Vol. 4, Issue 11, November 2015

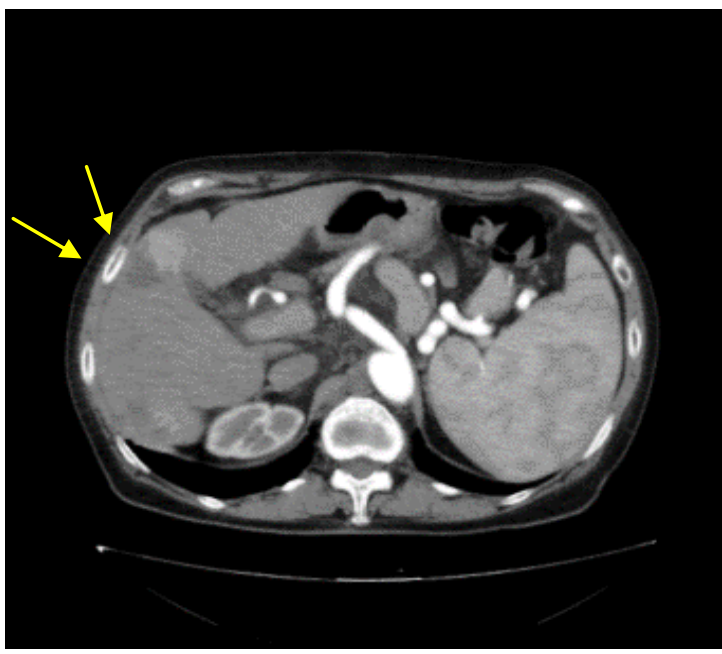

(a)

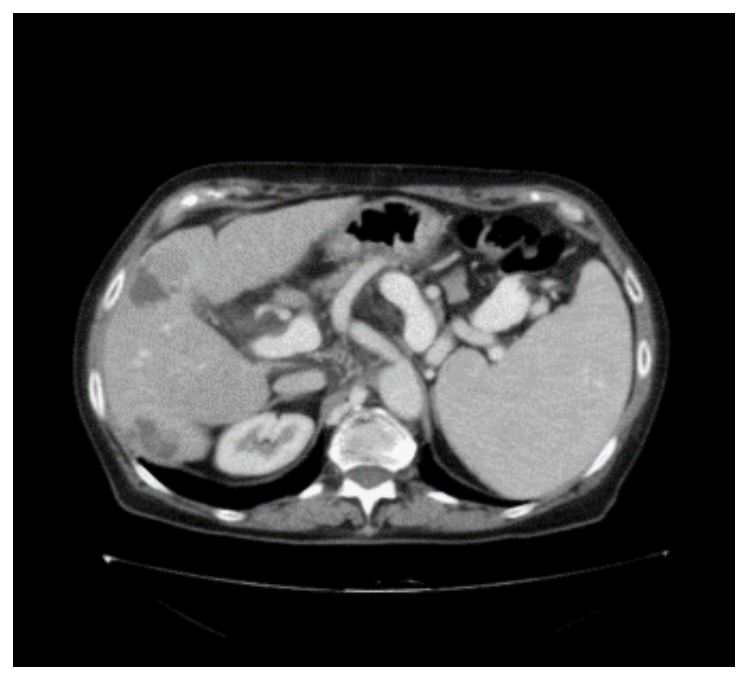

(b)

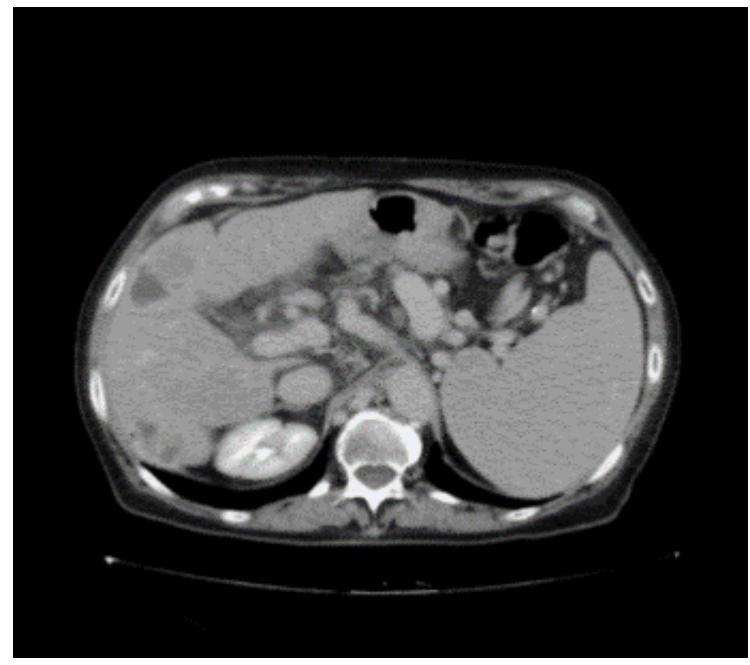

(c)

Fig. 1 Correspondent slices of triphasic CT, patient 2: (a) arterial phase, (b) portal-venous phase, (c) delayed phase

discriminant features, is perfectly consistent with the performance analysis described in paper [6], which was based on the optimization of quantitative color distance metrics.

\section{TISSUE CHARACTERIZATION}

A. Training Triphasic CT Dataset

Abdominal enhanced CT volumes (3 phases) have been taken with 512 by 512 pixels size and 8-bit grey-level from 7 patients affected by HCC tumor. The position, size and extent of tumors were defined in CT images by an expert radiologist. In each phase, for each patient, 5 slices, which better show the situation of tumor, were selected. In the liver two classes can be distinguished, healthy class and pathological class. In each slice, we took one window from the healthy part and one window from the pathological part.

The case study shown in Fig. 1 refers to patient p2 and deals with a HCC tumor after chemoembolization. The presence of a known sign in S8/S4 liver segments $(45 \times 33 \times 48 \mathrm{~mm})$ should be underlined. Such a formation has an inhomogeneous appearance as it is characterized in part by necrotic hypodense tissue and in part by neoplastic persistent tissue, as shown respectively with the arrows in Fig. 1a. The former is free from contrastographic enhancement. The latter shows some strengthening in arterial phase and moderate washout, as compared to neighboring parenchyma in venous and delayed phases. The statistical analysis for this case is reported as p2 and p2-treated as follows.

According to article [7] a 13 by 13 pixels window size has been chosen as the one related to minimum classification error rate for HCC tumor. At the end, we obtained a dataset consisting of 80 samples (40 samples for the healthy part and 40 samples for the pathological part).

Let $\mathrm{I}(1), \mathrm{I}(2)$, and $\mathrm{I}(3)$ represent phase 1, phase 2 and phase 3 images, respectively. The apices $\mathrm{H}$ and $\mathrm{P}$ represent healthy and pathological class respectively.

\section{B. Steady-State Analysis}

From a previous work [8] dealing with feature ranking and selection for lesion characterization from the same database, histogram-based metrics proved to stand up when compared with textural features. Specifically, position descriptors like first-order statistical moment and median values have always a great discriminant power. Based on such results, it was decided to directly apply the statistical analysis on local average values.

By looking independently at the mean value of each phase some common rules have been obtained as a meaningful result in the tissue characterization. Figures 2, 3, and 4 synthetically show what has been achieved for phase 2 , phase 3 , and phase 1 respectively.

\section{-}

$$
\text { RULE 001: } \mathrm{I}^{\mathrm{H}}(2)>\mathrm{I}^{\mathrm{P}}(2)
$$

Phase-2 Average Grey Level in Healthy class is greater than in Pathology class

This rule shows that when the majority of contrast agent is flowing through the hepatic portal vein the healthy part becomes lighter than the pathology part.

As explained above, this is the well-known washout phenomenon and is the reason why the tumor region is darker than the adjacent liver. In phase 2 this difference reaches large values (around 30). 


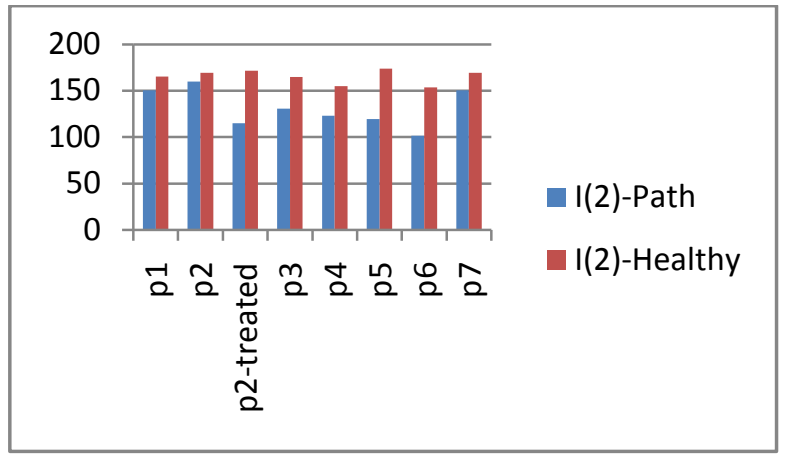

Fig.2 Average grey level in phase 2 for pathology and healthy samples

- RULE 002: $\mathrm{I}^{\mathrm{H}}(3)>\mathrm{I}^{\mathrm{P}}(3)$

Phase-3 Average Grey Level in Healthy class is greater than in Pathology class

One can observe that HCC tumor remains darker than the healthy part, showing again the negative enhancement effect. For all patients the healthy part is brighter than the pathology part. As compared with phase 2, the contrast between healthy and pathological tissues in phase 3 is much larger on an average.

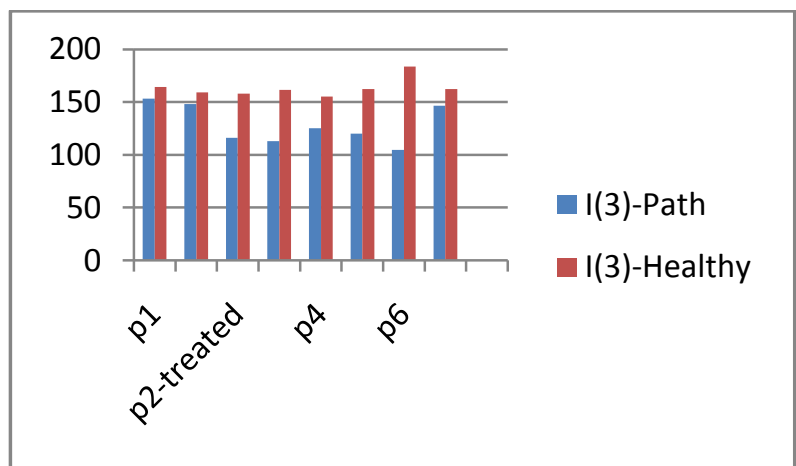

Fig.3 Average grey level in phase 3 for pathology and healthy samples

This rule shows that the best phase for a discriminant analysis of HCC tumor and healthy part, when the possible lesion has to be detected, is phase 3. At the same time, when shape and lesion border need to be detected, phase 3 seems the best volume to analyze thanks to the large image gradient. According to our previous work in paper [8], the classification error rate is the lowest when exploiting phase 3 .

\section{- $\quad$ Phase 1}

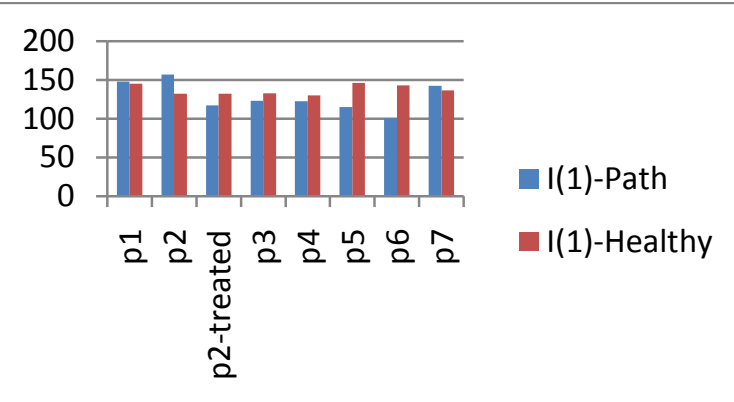

Fig.4 Average grey level in phase 1 for pathology and healthy samples
No common rule can be found when considering all patients and comparing healthy and pathological part from phase 1 volumes. According to a differential diagnosis, one can see that such a finding could discriminate between various pathology types (for instance early HCC, or plastic nodules, or atypical HCC). A scope which is not included in the present work.

\section{Temporal Statistical Analysis}

When multitemporal acquisitions are available the analysis can greatly benefit from a dynamic (i.e., temporal) feature analysis. The three phases for all patients are reported in Figures 5 and 6 for liver and pathological tissues, respectively.

By exploring such a kind of information, we can extract the following rules.

For healthy part (see Fig.5):

$$
\text { RULE 003: } \mathrm{I}^{\mathrm{H}}(2)>\mathrm{I}^{\mathrm{H}}(1)
$$

Mean Grey Level phase $2>$ Mean Grey Level phase 1 As expected, this rule shows that, for all patients and for all samples, the liver is enhanced in portal venous phase (phase 2) more than in arterial phase (phase 1). This is because the liver absorbs the contrast agent (c.a.) mainly through the hepatic portal vein.

- $\quad$ RULE 004: $\mathrm{I}^{\mathrm{H}}(3)>\mathrm{I}^{\mathrm{H}}(1)$

Mean Grey Level phase 3 > Mean Grey Level phase 1.

The same rule also holds for phase 3 when compared with phase 1.

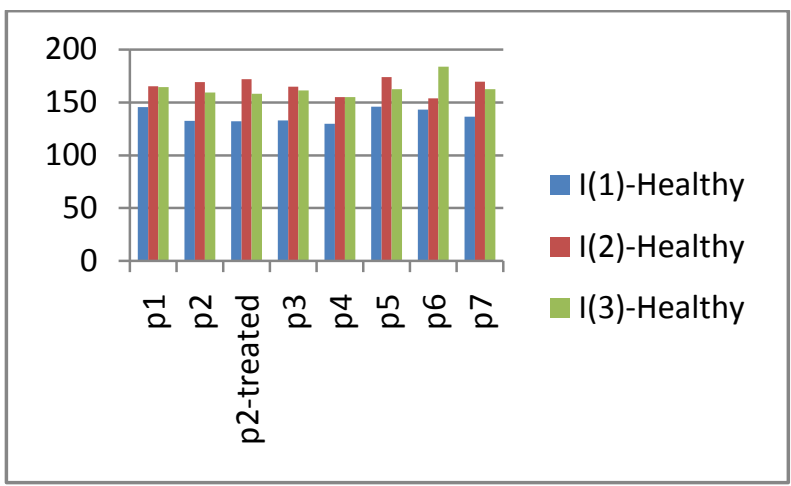

Figure5. Dynamic features in healthy samples

For HCC pathological regions (see Fig. 6):

RULE 005: $\mathrm{I}^{\mathrm{P}}(1)=\mathrm{I}^{\mathrm{P}}(2)=\mathrm{I}^{\mathrm{P}}(3)$

Mean Grey Level almost equal for all phases.

As can be seen in figure 3, the average grey level does not change significantly among the different phases.

Given i, $\mathrm{k}$ indexes of a CT phase, the general definition of a dynamic feature is achieved through the temporal gradient:

$$
\mathrm{m}_{\mathrm{ik}}=\mathrm{I}(\mathrm{i})-\mathrm{I}(\mathrm{k})
$$

As a major remark, it comes out that all the lesions show a very limited change from one phase to another, despite the perceptual impression given by a visual comparison. For instance, when looking to Fig.1, the rightmost lesion (hyperdense HCC) has exactly the same shade of grey in all three phases. 
International Journal of Advanced Research in Computer and Communication Engineering

Vol. 4, Issue 11, November 2015

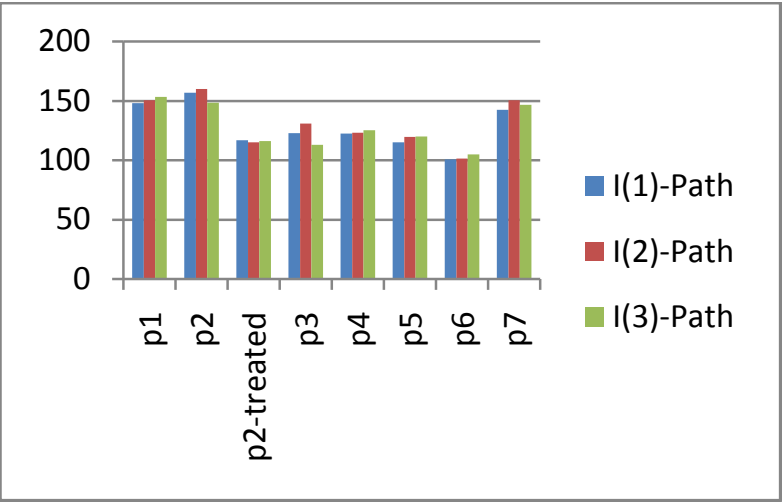

Figure6. Dynamic features in pathological samples

However, in the second and third phase it visually appears to be darker. This is the well-known Simultaneous Contrast [9] vision illusion and is due to a background being darker in the first phase than in the other ones.

At the same time, by comparing Fig.5 and Fig.6, one can see that the largest gradient in temporal analysis is the one related to healthy tissue.

The two above facts (corresponding to Rules 003 to 005) describe a different dynamic evolution of healthy and pathological regions, thus proving that the informative content of temporal features is extremely important.

\section{III.COLOR DATA FUSION}

Through the color data-fusion process the most relevant information content of each source image can be combined to form a more meaningful image, where various tissues are visible with different colors [10].

Based on the training set, this work proposes how to generate visualization able to improve the localization and identification of the pathology. The statistical analysis shows how all patients might benefit from such a procedure, since the features characterizing tissues are common to different individuals.

In order to visually enhancing in a single image the pathology with respect to healthy tissue, static and dynamic features can be exploited at the same time, as described in the following paragraphs. Instead of a direct association of each color RGB channel to one of the original source images, a functional association is proposed. Given that $f_{R}, f_{G}, f_{B}$ are specific scalar functions, a color image is generated by the following criterion as proposed in [5]:

$$
\left\{\begin{array}{l}
R=f_{R}(I(1), I(2), I(3)) \\
G=f_{G}(I(1), I(2), I(3)) \\
B=f_{B}(I(1), I(2), I(3))
\end{array}\right.
$$

The functionals $f_{R}, f_{G}, f_{B}$ can also take into account specific discriminating features or might directly correspond to the three best discriminant features as derived from a statistical analysis.

Specifically, in the present application, given that i,h,k,n, are indexes of a CT phase, it was decided to simplify the used color association functionals as follows:

$$
\left\{\begin{aligned}
f_{R} & =I(h) \\
f_{G} & =I(n) \\
f_{B}=m_{i k} & =I(i)-I(k)
\end{aligned}\right.
$$

Thus, two original sources are exploited (steady-state features) together with one temporal feature.

By focusing on the specific case study of liver tumor in Fig. 1, the best option of indexes i,h,k,n, in equation 3 is found based on the best steady-state and dynamic measurements.

Two criteria are used to evaluate the quality of the results displayed. The former is based on a perceptual ranking given by medical experts and non-medical individuals. The latter is based on the objective quantitative metrics related to some of the most recent color difference measures [6].

\section{IV.EXPERIMENTAL WORK}

For a more comprehensive description, the case study of patient 2 is described in detail as follows. Here the tumor and the necrotic parts are taken into account at the same time.

As expected, Rules 001 and 002 hold for this case, since healthy part is lighter than pathological parts both in phase 2 and 3, as pointed out in Fig.7, thanks to HCC washout and the null absorption of necrotic mass. Thus, it is clear that informative contents related to Phase 2 and Phase 3 are strongly directly correlated so that even only one of such phases is sufficient.

On the contrary, the same rule does not hold for Phase 1. In fact, there is a contrast inversion between liver and hyper dense mass when comparing Phase 1 with Phase 2 or 3. The treated tissue (leftmost lesion in Fig.1) is very insensitive to c.a. and is always darker than the liver. Phase 1 carries a lot of information and is obviously selected as one contributing to the color image.

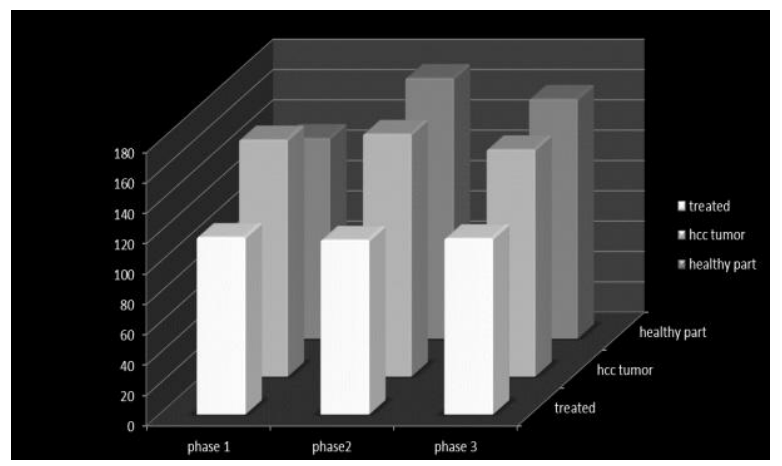

Fig.7. Necrotic tissue, HCC tumor, and Liver of patient 2

When studying the dynamical progress, the difference between phases is analysed and reported in Fig.8. Even though the absolute difference of liver and tumor when looking to $\mathrm{m}_{21}$ is larger, the different sign observed in feature $m_{31}$ is much more meaningful and represents an optimal descriptor for tissue discrimination. For this same feature, both necrotic and tumoral tissues have a large spread. 
International Journal of Advanced Research in Computer and Communication Engineering

Vol. 4, Issue 11, November 2015

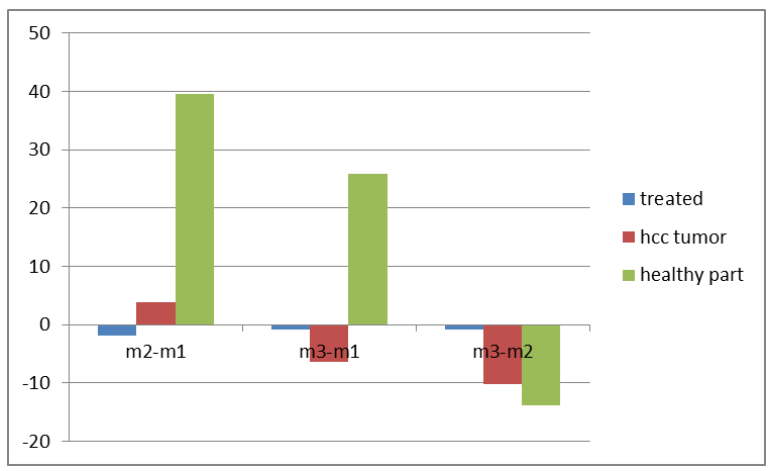

Fig.8 Dynamic features for necrotic tissue, HCC tumor, and Liver of patient 2

The final decision has then been to apply the following color association rule:

$$
\left\{\begin{array}{c}
f_{a}=I(1) \\
f_{b}=I(3) \\
f_{c}=I(1)-I(3)
\end{array}\right.
$$

where the notation $\mathrm{fa}, \mathrm{fb}, \mathrm{fc}$ allows to change the order of functionals $f_{R}, f_{G}, f_{B}$ to achieve different permutations. In Fig.9 some examples are shown. As one can see, the color allows the tumor masses to better stand out from the parenchyma.

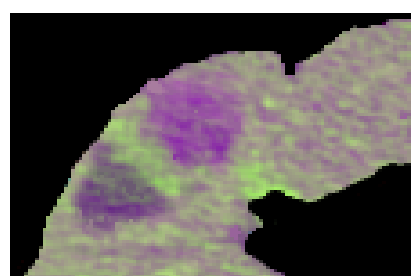

(a)

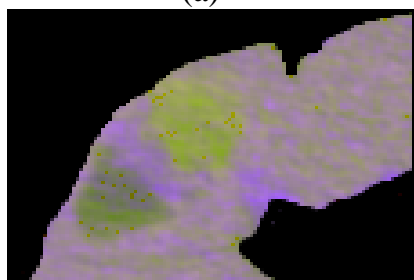

(b)

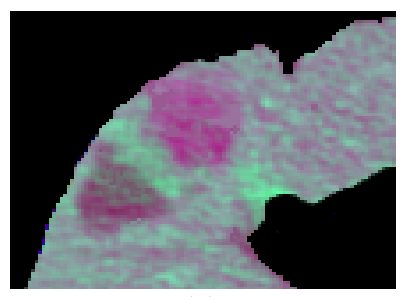

(c)

Fig.9. Proposed color data-fusion result with a few permutations

The reader is referred to [6] for a quantitative evaluation of the generated data-fusion images. The result here proposed, obtained through statistical considerations, is perfectly consistent with the one proposed in the above paper. In particular, it holds also in the present case that the best quality is associated with the best color contrast metrics as evaluated by Lab, Luv, CMC (and RGB) distance measures.

\section{CONCLUSION}

The feature analysis performed on real cases confirmed in a quantitative way that lesion vascularity is a major finding in liver pathologies and has also shown how human visual perception may be sometimes misleading. The aim of this work was not to obtain a classification, but to improve image visualization and demonstrate how image details can be immediately highlighted by an appropriate use of colors. The use of the newly proposed color fusion method allows obtaining a single integrated color image, able to highlight small details from triphasic CT volumes. Moreover, this method can also be applied to other multi-temporal and multi-parametric images and volumes.

\section{REFERENCES}

[1] F. Edward Boas, Aya Kamaya, Bao Do, Terry S. Desser, Christopher F. Beaulieu, Shreyas S. Vasanawala, Gloria L. Hwang, Daniel Y. Sze, "Classification of Hypervascular Liver Lesions Based on Hepatic Artery and Portal Vein Blood Supply Coefficients Calculated from Triphasic CT Scans," Digital Imaging, vol.28, pp.213-223, 2015.

[2] Deepak Bhayana,Tae Kyoung Kim, Hyun-Jung Jang, Peter N Burns, Stephanie R. Wilson, "Hypervascular Liver Masses on Contrast-Enhanced Ultrasound: The Importance of Washout," AJR Am J Roentgenol., vol.194(4), pp.977-83, Apr 2010.

[3] Nils Albiin, "MRI of Focal Liver Lesions," Current Med Imaging vol. 8(2), pp.107-116, May 2012.

[4] R. Lencioni, L. Crocetti,M.C. Della Pina, D. Cioni, "Guidelines for imaging focal lesions in liver cirrhosis," Expert Rev Gastroenterol Hepatol., vol. 2(5 ), pp. 697-703, 2008.

[5] S.Dellepiane, I.Minetti, G. Vernazza, "Method for the extraction of information of interest from multi-dimenisional, multi-parametric and / or multi-temporal datasets," United States Patent Application 20130343637, 2013.

[6] R. Ferretti and S. Dellepiane, "Color Spaces in Data Fusion of Multi-Temporal Images," ICIAP 2015, Springer-Verlag Berlin Heidelberg.

[7] D. Smutek, A. Shimizu, H. Kotake, S. Nawano, L. Tesar, "Texture Analysis of Hepatocellular Carcinoma and Liver Cysts in CT images," in SPPRA, pp.56-59, 2006.

[8] Mahdieh Khalilinezhad, Silvana Dellepiane, Gianni Vernazza, "Detecting HCC tumor in three phasic CT liver image with optimization of neural network," Int. Journal of Medical, Health, Biomedical and Pharmaceutical Engineering, World Academy of Science, Engineering and Technology, vol.9 (3), pp. 262-267, 2015.

[9] Sidney Stecher, "Luminance-Difference Thresholds and Simultaneous Contrast," The American Journal of Psychology, Vol. 81 (1), pp. 27-35, Mar. 1968.

[10] S.G. Dellepiane, E. Angiati, “A new method for cross-normalization and multitemporal visualization of SAR images for the detection of flooded area,", IEEE Transactions on Geoscience and Remote Sensing, vol. 50(7), pp. 2765-2779, 2012.

[11] R. Joel Gibson, "Spiral CT of the Liver: is Biphasic or Triphasic Scanning the Routine in your Department?," Advance for Imaging \& Radiation Oncology, 2010.

\section{BIOGRAPHIES}

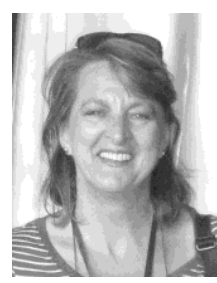

Silvana G. Dellepiane graduated in 1986 with honours. In 1990 she received the $\mathrm{PhD}$ degree in Electronic Engineering and Computer Science. In 1992 she became a Researcher (Assistant Professor) in the Department of Biophysical and Electronic Engineering (DIBE), Università degli Studi di Genova, 
where she is an Associated Professor in the biomedical field. She is co-author of papers presented at Telecommunication area, belonging to DITEN international conferences. She is also IEEE Student department. She has taught in Signal Theory and Pattern Member associated to IEEE Signal Processing Society.

Recognition. At present she is professor of Electrical Communications, Statistical Methods, Signal and Image Processing and Recognition, in the courses of Telecommunications Engineering and Bioengineering. She is responsible for the NUMIP research area of the Signal Processing and Telecommunications (SP\&T) laboratory at DITEN. Prof. Dellepiane has gained wide scientific and technical experience in multi-dimensional data processing. Her main research interests include the use of context and fuzzy systems for multi-dimensional data processing, segmentation, supervised methods for the processing of remote sensing SAR images, and non-linear adaptive processing of digital signals. Her application domains are, mainly, telemedicine and remote sensing. Silvana Dellepiane is a reviewer for various journals. She was invited at some international conferences and schools for tutorials and lessons. She has participated, at the organization and scientific levels, in the research activities concerning various CNR, ASI, MIUR and UE projects. She has been a member of the Technical Committee "Bio Imaging and Signal Processing" of IEEE Signal Processing Society.

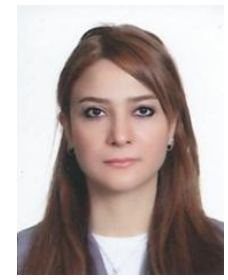

Mahdieh Khalilinezhad got her bachelor on Applied Mathematics in 2005 From Azad University-Tehran Markazi Branch in Tehran. She got her Master in Information Technology Engineering from university of Qom in Iran at 2010. Since January 2013 up to now, she is $\mathrm{PhD}$ student in Computational Intelligence, University of Genova in Italy. She has several papers in international conferences and journals in the field of Image Processing, Pattern Recognition and Data Mining areas. Her current researches include Neural Network and their application to Biomedical Image in CT/ MR image analysis. Her interest areas include $2 \mathrm{D}$ and $3 \mathrm{D}$ feature selection, feature reduction, uses of fuzzy systems for supervised and unsupervised data. Her application of work is about Biomedical Images and Satellite Images.

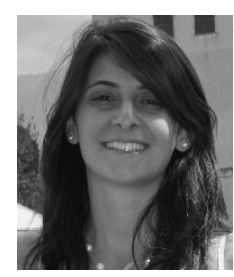

Roberta Ferretti was born in Genova, Italy, on May 08, 1987. She obtained the Bachelor's degree (BSc) in March 2010 and the Master degree (MSc) in July 2012 both in Biomedical Engineering at University of Genoa. Since January 2013 she has been cooperating with NUMIP area of the Signal \& Image Processing group at DITEN (Department of Naval Electrical Electronical and Telecommunication Engineering) in research activities concerning Image Processing and Data Fusion algorithms. Since January 2014 she is a Ph.D. student in Science and Technology for Electronics and Telecommunication Engineering: her research topics are techniques of nonlinear processing, segmentation, data fusion and analysis of the quality of digital images. The application domain is 